\title{
Pine Bark Extract Protects Against Oxidative Stress Induced By Recurrent Hypoglycemia In Rats.
}

\author{
Mamdouh Rashad Farag El-Sawi \\ (Zoology Department, Faculty of Science, Mansoura University, Mansoura, Egypt)
}

\begin{abstract}
The present work was designed to evaluate the role of pine bark extract (PBE) in protecting rats from oxidative stress produced by recurrent hypoglycemia. The animals were grouped as the following: control group, PBE-treated group (treated with $150 \mathrm{mg} / \mathrm{kg} \mathrm{b} \mathrm{wt}$; daily via oral gavage for 30 consecutive days); recurrent hypoglycemia group (injected with 1.14 units of insulin/ $\mathrm{kg} b$ wt; day after day subcutaneously, for 30 days) and recurrent hypoglycemia \& PBE-treated group (the rats received PBE and insulin as in group 2 and group 3, respectively). Serum glucose and total proteins (TP) as well as liver and brain glycogen contents were significantly decreased when compared with both control and PBE-treated groups but, they were increased significantly when compared with recurrent hypoglycemia group.Whereas serum insulin and $\gamma$-Glutamyl transferase $(\gamma-G T)$ activity were increased when compared with both control and PBE-treated groups. Liver and brain total antioxidant capacity (TAC) as well as, superoxide dismutase (SOD), catalase (CAT) and glutathiones-transferase (GST) activities were significantly decreased when compared with both control and PBE-treated groups. Whereas, malonedialdehyde (MDA) levels of both liver and brain tissues were increased significantly when compared with both control and PBE-treated groups. Liver glutathione (GSH) content showed significant decrease whereas, brain GSH showed insignificant change.
\end{abstract}

Keywords: Oxidative stress; recurrent hypoglycemia; Pine bark extract; Insulin; Rats.

\section{Introduction}

The balance between harmful and beneficial effects of free radicals are very important for living organisms [1]. Recurrent Hypoglycemia is achieved due to increased insulin doses for patients with diabetes type I or may be as a result of a congenital deformity of the metabolic processes or birth defects in insulin secretion for people who have diabetes. Several previous studies showed that hypoglycemia can cause oxidative stress by increasing reactive oxygen species (ROS) which caused brain tissue injury and death [2] [3] [4] [5] or by increasing the production of lipid peroxidation products (LPO) such as MDA [6] [7] [8] and the deficiency in the activity of SOD as well as the depletion of GSH content in the crust and hippocampus areas of the brain of mice [6] [8]. Pine bark extract (PBE) has many phenolic compounds such as catechins and epicatechins with beneficial impacts for human health.PBE from the French maritime pine tree (Pycnogenol, PY); Pinus pinaster. PY was described as a dietary supplement in many countries especially the United States as it is very safe. All its components are involved in and work to disable free radicals and stimulates body cells to duplicate antioxidants production in addition, they neutralize directly the free radicals [9] [10] [11].

Pine bark extract can increase the activity of endogenous antioxidants such as GSH, SOD, and catalase (CAT). Giving PY for children with attention deficit hyperactivity disorder (ADHD) significantly decreased oxidized glutathione (GSSG), increased GSH, and improved ratio of GSH/ GSSG as well as the TAC level was restored to the normal level [12]. Polyphenols of PY increased the synthesis of zinc and copper SOD significantly and decreased $\gamma$-GT activity in the blood of diabetic rats to the normal level [13]. French PBE (PY) protected against oxidative stress caused by Cisplatin in the bone marrow, kidney and red blood cells in the white rats. PY significantly ameliorated MDA level and nitric oxide to the normal level, as well as increased antioxidant enzymes activity and they suggested that PY has a protective effect against the toxicity associated with treatment with Cisplatin [14]. Pine bark extract protected against oxidative damage induced by chromium toxicity in rat's skin. The results obtained showed that PBE reduced the level of LPO, while it increased CAT and GST activity [15].

Many of the laboratory and clinical studies supported the protective role of PY to control diabetes. PY significantly reduced the level of MDA and protein carbonyl (PC) to the normal level, as well as it maintained GSH and GST and CAT activity in liver tissue to the normal range [16] The lipids, proteins and DNA are the basic targets of oxidative damage and the inhibition mechanism of the LPO by pine bark of Pinus pinaster; PY included direct removal of free radicals, especially $\mathrm{OH}^{*}$ and $\mathrm{O}_{2}{ }^{-}$. Studies have shown that PY can prevent oxidative damage to lipids and proteins [17]. PY is used in medical conditions induced by oxidative stress. The present study aimed to test the ability of PBE to protect body cells from oxidative stress status that results from recurrent hypoglycemia in male albino rats [18]. 


\subsection{Chemicals}

\section{Materials And Methods}

Pine bark extract was obtained from Swanson Health Company, USA. Insulin used is manufactured by SEDICO Pharmaceutical Co and was obtained from local pharmacy. All other chemicals were purchased ready as kits from Bio-diagnostic Company, Cairo, Egypt.

\subsection{Experimental animals}

Forty Wistar male rats were obtained from the VACCERA center in Helwan city, Helwan governorate, Egypt and used for the experiment; their weights range between 120 and $140 \mathrm{~g}$. They were remained for a week before the start of the experiment for acclimatization. They were caged under healthy conditions and light: darkness cycle (12 hours light - 12 hours of darkness). Water was available for the whole duration of the experiment.

\subsection{Experimental design}

The rats were divided randomly into four groups (10 rats for each group) as follows: Group I: served as control; they did not receive any treatment. Group II: PBE-treated group; they were treated (orally by stomach tube) with a dose of $150 \mathrm{mg}$ PBE/ kg b wt.; daily for 30 consecutive days. Group III: group of insulin-treated (the case of recurrent hypoglycemia) they were injected with a dose of 1.14 units of insulin / $\mathrm{kg} b$ wt.; day after day subcutaneously, for 30 days. Group IV: insulin and PBE- treated group; they were injected with PBE as in group II, as well as insulin, as in group III.

\subsection{Blood sampling}

At the end of the experiment, the overnight fasted animals were anesthetized by ether, dissected quickly and by using a syringe, blood sample was withdrawn directly from the ventricle and then put in centrifuge tube and left for 20 minutes until full blood clot (at room temperature) and then centrifuged at 3000 rpm for 15 min. Blood serum was separated using a dropper of Pasteur, a volume of serum was directly used for measuring glucose and the rest of the serum sample was stored in the refrigerator at a temperature of $-20 \mathrm{C}^{\circ}$ until making the rest of biochemical analyses later.

\subsection{Liver and brain tissue homogenates preparation}

A weight of each liver or brain was used, then placed in a dry and clean centrifuge tube, grinded by homogenizer in phosphate buffer solution to prepare a $10 \%$ weight/ volume homogenate. It was centrifuged and supernatant was separated for each sample (except that for GSH determination); using micropipettes, put in clean and dry Eppendorf tubes, then labelled and stored at $-20^{\circ} \mathrm{C}$ for subsequent analyses.

\subsection{Methods}

All parameters were assessed by using assay kits from Bio-diagnostic Company, Cairo, Egypt. Insulin was determined using ELISA technique. Glucose, Glycogen, GSH, GST, CAT, SOD, TAC, $\gamma$-GT, MDA and TP were determined according to the methods of [19], [20], [21], [22], [23], [24], [25], [26], [27] and [28], respectively.

\subsection{Statistical analyses}

Statistical methods that have been followed in the analyses of the data were 1.Descriptive methods; using the arithmetic mean and standard error. 2. Deductive methods; using: A) One-way analysis of variance (ANOVA) for comparison between the different groups. B) Least Significant Difference (LSD) was used when there was a difference between the groups.

\section{Results}

Table (1): Biochemical changes in control and different treated rat groups.

\begin{tabular}{|c|c|c|c|c|}
\hline Rat Groups & Control & PBE & Recurrent & Recurrent \\
\hline & & & Hypoglycemia & Hypoglycemia \\
\hline Parameters & & & & $+\mathrm{PBE}$ \\
\hline \multirow[t]{2}{*}{ Serum Insulin (U/L) } & $9.8 \pm 0.20$ & $9.8 \pm 0.20$ & $15.8 \pm 0.14^{0^{\circ}}$ & $12.7 \pm 0.13^{0 * x}$ \\
\hline & & {$[-0.27]$} & {$[60.1]$} & [29.3] \\
\hline \multirow[t]{2}{*}{ Serum Glucose (mg/ dl) } & $92.5 \pm 2.23$ & $94.2 \pm 3.78$ & $56.8 \pm 1.36^{\circ}$ & $70.4 \pm 2.49^{0-x}$ \\
\hline & & [1.91] & {$[-38.6]$} & {$[-23.9]$} \\
\hline \multirow[t]{2}{*}{ Liver Glycogen (mg/ $100 \mathrm{~g})$} & $30.5 \pm 0.49$ & $30.6 \pm 0.61$ & $24.2 \pm 0.690^{\circ}$ & $27.5 \pm 0.28^{\circ \cdot x}$ \\
\hline & & [0.18] & {$[-20.6]$} & {$[-9.90]$} \\
\hline \multirow[t]{2}{*}{ Brain Glycogen (mg/ 100g) } & $11.09 \pm 0.2$ & $11.09 \pm 0.18$ & $10.56 \pm 0.12^{\circ}$ & $10.9 \pm 0.14$ \\
\hline & & {$[-]$} & {$[-4.78]$} & {$[-1.66]$} \\
\hline \multirow[t]{2}{*}{ Serum $\gamma$ - GT (U/L) } & $33.7 \pm 1.39$ & $31.5 \pm 1.24$ & $89.4 \pm 1.290^{\circ}$ & $62.5 \pm 1.33^{0-x}$ \\
\hline & & {$[-6.44]$} & {$[-53.51]$} & {$[-14.15]$} \\
\hline Serum TP (g/ dl) & $7.05 \pm 0.12$ & $7.06 \pm 0.13$ & $5.56 \pm 0.16^{\circ}$ & $6.66 \pm 0.1^{-x}$ \\
\hline & & [0.142] & {$[-21.13]$} & \\
\hline
\end{tabular}

$[-5.53]$

Values are mean \pm SE of 10 samples. Least significant difference (LSD) between groups are considered significant at $\mathrm{P} \leq 0.05 .^{\circ}=$ significant with control, ${ }^{\circ}=$ significant with $\mathrm{PBE}$ and ${ }^{\times}=$significant with recurrent hypoglycemia group. Values between parentheses are $\%$ of change from control. 
Table (1) showed that serum glucose, TP as well as liver and brain glycogen contents were significantly decreased when compared with both control and PBE groups but, they were increased significantly when compared with recurrent hypoglycemia group. Whereas serum insulin level and $\gamma$ - GT activity were increased when compared with both control and PBE groups but, they were decreased significantly when compared with recurrent hypoglycemia group. Only the brain glycogen and the TP have reached the normal range due to PBE treatment.

Table (2): Liver oxidative stress markers in control and different treated rat groups.

\begin{tabular}{|c|c|c|c|c|}
\hline Rat Groups & Control & PBE & Recurrent & Recurrent \\
\hline & & & Hypoglycemia & Hypoglycemia \\
\hline Parameters & & & & + PBE \\
\hline \multirow[t]{2}{*}{ MDA (nM/g) } & $5.40 \pm 0.23$ & $2.76 \pm 0.25^{\circ}$ & $12.43 \pm 0.33^{\circ *}$ & $0.22^{0 * x}$ \\
\hline & & {$[-48.86]$} & [129.88] & [56.89] \\
\hline \multirow[t]{2}{*}{ GSH (mg/g) } & $18.9 \pm 0.34$ & $19.7 \pm 0.24$ & $17.7 \pm 0.38^{\circ}$ & $=0.22^{\circ}$ \\
\hline & & [4.35] & {$[-5.91]$} & {$[-4.17]$} \\
\hline \multirow[t]{2}{*}{$\mathrm{CAT}(\mathrm{U} / \mathrm{g})$} & $2.26 \pm 0.26$ & $2.29 \pm 0.13$ & $0.88 \pm 0.04^{\circ}$ & $0.13^{x}$ \\
\hline & & [1.5l] & {$[-60.9]$} & {$[-31.6]$} \\
\hline \multirow[t]{2}{*}{ SOD (U/g) } & $1119 \pm 16.7$ & $1284 \pm 74^{\circ}$ & $549 \pm 22^{\circ}$ & $\pm 25^{\circ x}$ \\
\hline & & [14.74] & {$[-50.9]$} & {$[-2.56]$} \\
\hline \multirow[t]{2}{*}{ TAC (mM/g) } & $1.01 \pm 0.01$ & $1.04 \pm 0.018$ & $0.81 \pm 0.016^{\circ}$ & $1.0 \pm 0.012$ \\
\hline & & [2.97] & [-19.49] & {$[-1.08]$} \\
\hline GST (U/g) & $12.8 \pm 0.19$ & $13.3 \pm 0.25$ & $7.16 \pm 0.1^{0^{*}}$ & $11.03 \pm 0.29^{\circ * x}$ \\
\hline$[-13.59]$ & & [4.41] & {$[-43.89]$} & \\
\hline
\end{tabular}

Values are mean $\pm \mathrm{SE}$ of 10 samples. LSD between groups are considered significant at $\mathrm{P} \leq 0.05$. $^{\circ}=$ significant with control, ${ }^{\bullet}=$ significant with PBE and ${ }^{\times}=$significant with recurrent hypoglycemia group. Values between parentheses are $\%$ of change from control.

Table (2) showed that liver GSH, TAC contents as well as liver CAT, SOD GST activities were significantly decreased when compared with both control and PBE groups but, they were increased significantly when compared with recurrent hypoglycemia group. Whereas MDA was increased significantly when compared with both control and PBE groups but, it was decreased significantly when compared with recurrent hypoglycemia group. Most parameters (GSH, SOD \& TAC) have reached the normal range due to PBE treatment.

Table (3): Brain oxidative stress markers in control and different treated rat groups.

\begin{tabular}{|c|c|c|c|c|}
\hline Rat Groups & Control & PBE & Recurrent & Recurrent \\
\hline & & & Hypoglycemia & Hypoglycemia \\
\hline Parameters & & & & $+\mathrm{PBE}$ \\
\hline \multirow[t]{2}{*}{ MDA (nM/g) } & $3.59 \pm 0.44$ & $2.60 \pm 0.23$ & $9.27 \pm 0.31^{0^{*}}$ & $5.68 \pm 0.14^{0 * x}$ \\
\hline & & {$[-27.7]$} & [157.8] & [57.8] \\
\hline \multirow[t]{2}{*}{ GSH (mg/g) } & $18.1 \pm 0.30$ & $18.8 \pm 0.42$ & $18.1 \pm 0.21$ & $18.2 \pm 0.23$ \\
\hline & & [3.65] & {$[-0.48]$} & {$[0.43]$} \\
\hline \multirow[t]{2}{*}{$\mathrm{CAT}(\mathrm{U} / \mathrm{g})$} & $1.40 \pm 0.078$ & $1.67 \pm 0.075^{\circ}$ & $0.64 \pm 0.017^{\circ}$ & $1.15 \pm 0.046^{0 \cdot x}$ \\
\hline & & [18.82] & {$[-54.5]$} & {$[-18.32]$} \\
\hline \multirow[t]{2}{*}{ SOD (U/g) } & $1121 \pm 20.3$ & $1145 \pm 23.9$ & $671 \pm 31^{\circ}$ & $1070 \pm 29.9^{\times}$ \\
\hline & & [2.103] & {$[-40.13]$} & [4.59] \\
\hline \multirow[t]{2}{*}{ TAC (mM/g) } & $0.81 \pm 0.029$ & $0.92 \pm 0.034$ & $0.38 \pm 0.072^{\circ}$ & $0.70 \pm 0.028^{* x}$ \\
\hline & & [23.03] & {$[-76.3]$} & {$[-14.03]$} \\
\hline \multirow[t]{2}{*}{ GST (U/g) } & $8.01 \pm 0.116$ & $8.28 \pm 0.187$ & $3.30 \pm 0.095^{\circ}$ & $5.46 \pm 0.134^{0 \cdot x}$ \\
\hline & & {$[3.40]$} & {$[-58.80]$} & \\
\hline
\end{tabular}

Values are mean \pm SE of 10 samples. LSD between groups are considered significant at $\mathrm{P} \leq 0.05 .^{\circ}=$ significant with control, ${ }^{\cdot}=$ significant with PBE and ${ }^{\times}=$significant with recurrent hypoglycemia group. Values between parentheses are $\%$ of change from control.

Table (3) showed that brain TAC content as well as liver CAT, SOD GST activities were significantly decreased when compared with both control and PBE groups but, they were increased significantly when compared with recurrent hypoglycemia group. MDA was increased significantly when compared with both control and PBE groups but, it was decreased significantly when compared with recurrent hypoglycemia group. GSH showed insignificant changes when compared with all other groups. Only the SOD activity and TAC have reached the normal range due to PBE treatment. 


\section{Discussion And Conclusion}

Hypoglycemia (Glucose level $<60 \mathrm{mg} / 100 \mathrm{ml}$ ) is caused by congenital or acquired reasons. Persons with diabetes are much more susceptible to the condition of hypoglycemia due to drugs which increases the activity of insulin secretion and reduces the blood glucose level. The most common forms of hypoglycemia occur as a complication of diabetes with insulin or hypoglycemic drugs for oral therapy. Serum glucose deficiency is less common in people without diabetes, but can occur at any age through excessive insulin production (cancer of islets of Langerhans), a hereditary and error in metabolism, drugs and toxins, alcohol, lack of certain hormones such as growth hormone and cortisol for long periods [29].

Reliance on what explained by [30] which indicated that hypoglycemia induced by insulin caused a decline in GSH and SOD in rat's brain, liver and kidney tissues; as well as the results of the study conducted by the [6] on healthy persons for the study of antioxidant activity in the case of hypoglycemia induced by insulin, where they concluded an increase in MDA content and increased $\gamma$-GT activity and a decrease in SOD activity as well as GSH level. Also, the results obtained by [2] indicated that hypoglycemia may cause an increase in oxidative stress in the brain tissue and a decrease in activity of SOD. Therefore, this study aimed to investigate the effect of the PBE on semantics oxidative stress; through follow-up of the activity of the enzymes CAT, SOD, GST and TAC, MDA contents, in homogenates of liver and brain of rats subjected to recurrent Hypoglycemia.The results of this study showed that treatment of rats with insulin only- the group of recurrent hypoglycemia- caused a significant decrease in serum glucose level and the TP content in blood and it was accompanied by a significant increase in insulin level and in serum $\gamma$-GT, compared to the control group. These results mean that the increase in insulin and frequent hypoglycemia has negative effects on the liver, where it decreased the TP content and also increased the liver enzymes activity. Decreased protein content may be due to significant elevation in insulin levels which stimulates body cells to take amino acids, which represent the basic units of proteins and to exploit these acids in the synthesis of proteins. Thus, any defect in insulin directly affects the growth process and increased liver enzymes activities can be explained by the significant increase in oxidative stress in the liver [31].

It also found that recurrent hypoglycemia due to insulin injection reduced unexpectedly, liver glycogen content significantly.While the decrease in the brain's glycogen wasn't significant. There was a significant decrease in the activity of antioxidant enzymes in the liver and the brain tissues, such as CAT, SOD and GST with a significant decrease in GSH and TAC, and on the contrary, there was a significant increase in MDA in the liver and brain tissues. These results confirm the increased oxidative stress in case of insulin-induced hypoglycemia and that the main reason for the increased oxidative stress in a patient with diabetes not only due to the increased glucose content, but also due to hypoglycemia.

The results of this study are concomitant with the results of the study carried out by the [2] which showed that hypoglycemia may cause an increase in oxidative stress in the brain tissue, and also with the study carried out by [8] which stated that hypoglycemia has led to an increase in the level of MDA with the decreased activity of SOD and GSH content of the brain's cortex and is also consistent with studies conducted by [32], [33], [34] and [35]. The accumulation of large quantities of zinc in neurons as a well as the result of decreased glucose level; has led to an increase in the production of ROS in the nerve cells which led to increased oxidative stress in the brain cortex and the hypothalamus and hypoglycemic coma and brain injury are complications. The potential for the treatment of insulin and oxidation resulting from the shortage of sugar is the main cause of death of the nerve cells [3].

The results of the present study showed that the treatment of normal rats with PBE only, significantly increased serum glucose and TP contents accompanied by an insignificant decrease in insulin level and in the hepatic $\gamma$-GT, when compared to the control group. These results suggested that the use of PBE in control rats had no effects on blood glucose level, insulin and TP and it improved the activity of liver enzymes and this can be explained on the basis that PBE protected the liver from oxidative stress [17] [18] [36].

It was also found that the use of PBE for normal rats did not affect the glycogen content in the liver and brain. On the contrary, it has been found that the use of PBEY increased the activity of antioxidant enzymes in the liver and the brain, such as the CAT of liver and brain, SOD of liver and this increase was statistically significant, while the PBE increased insignificantly the activity of GST, the content of GSH and TAC in the liver and brain tissues. On the other hand, the use of PBE in rats reduced the content of MDA in the liver and brain tissues. These results suggested that the use of PBE in rats improved the case of oxidative stress by increasing the activity of CAT, SOD and also by reducing the end products of oxidative stress of lipids membranes (MDA). These results indicated that the use of PBE improved liver functions and oxidative stress in the liver and brain tissues; through improving the activity of the enzymes CAT, SOD, GST and MDA content in rats treated only by PBE when compared to the control group. The results are compatible with that obtained by [9] [13] [14] [15] [16] [37] [38] [39]. The results of this study showed that treatment of rats with PBE and insulin has led to significant increase in glucose and protein content of the liver; this was accompanied by significant decrease in insulin and also in hepatic $\gamma$ - GT comparing with recurrent hypoglycemia group. These 
results indicated that the use of PBE in case of recurrent hypoglycemia improved glucose and liver functions. Improved liver functions can be interpreted according to the results of this study because of the improvement in oxidative stress of the liver. These results are consistent with the findings of [38] and [40] who recommended that, the use of PBE improved liver glycogen significantly, but in case of recurrent hypoglycemia the improvement was insignificant.

The results of the current study showed that PBE increased the activity of antioxidant enzymes in the liver and the brain, such as the CAT, SOD and GST with a significant increase in GSH content and in the total antioxidant capacity (TAC) and also there was a significant decrease in liver and brain MDA. These results suggested that improved state of oxidative stress as a result of the frequent lack of sugar through insulin injections was achieved by PBE treatment. The results obtained in the present study were consistent with that of [12] [14] [38] [40] on children for of GSH and TAC also with [15] for GSH, CAT and SOD, GST. Also coincided current findings regarding moral increase in the TP content in the animals treated group PBE and insulin only with the study conducted by [39], although the increase in the content of GSH in addition to increasing the activity of GST, CAT and SOD, which led to a rise in the TAC by treatment PBE due to the ability of cells to stimulate the production of components for most of the antioxidants in addition to the doubling of the cell's production of the SOD, CAT and the GSH, which represent the most powerful weapon to disable free radicals [41].

\section{Conclusion}

Recurrent hypoglycemia induced oxidative stress in rats and treatment with PBE showed an ameliorative effect against oxidative status produced, as it modulates most of tested parameters of oxidative stress to be close to the normal values.

\section{References}

[1]. W. Dröge, Free radicals in the physiological control of cell function. Physiol. Rev., 82(1), 2002, 47-95.

[2]. J. Patočkova, P. Marhol, E. Tumova, M. Krisiak, R. Rokyta, S. Stipek, J. Crkovska and M. Andel, Oxidative stress in the brain tissue of laboratory mice with acute post insulin hypoglycemia, Physiol Res., 52 (1), 2003, 131-135.

[3]. S. W. Suh, E. T. Gum, A. M. Hamby, P. H. Chan and R. A. Swanson, Hypoglycemic neuronal death is triggered by glucose reperfusion and activation of neuronal NADPH oxidase. J Clin Invest., 117 (4), 2007, 910-918.

[4]. J. L. Wayenberg and A. Pardou, Moderate hypoglycemia in the preterm infant: is it relevant? Arch Pediatr., 15 (2), $2008,153-156$.

[5]. X. Fioramonti, A. Deak, S. Deshpande, L. Carneiro, C. Zhou, N. Sayed, B. Orban, J. R. Berlin, L. Pénicaud, C. Leloup, A. Beuve and V. H. Routh, Hypothalamic S-nitrosylation contributes to the counter-regulatory response impairment following recurrent hypoglycemia. PLoS One, 8 (7), 2013, e68709.

[6]. J. Koska, P. Blazícek, M. Marko, J. D. Grna, R. Kvetnanský and M. Vigas, Insulin, catecholamines, glucose and antioxidant enzymes in oxidative damage during different loads in healthy humans. Physiol Res., 49 (1), 2000, S95-100.

[7]. M. L. Haces, T. Montiel and L. Massieu, Selective vulnerability of brain regions to oxidative stress in a non-coma model of insulininduced hypoglycemia. Neuroscience, 165 (1), 2010, 28-38.

[8]. S. Cardoso, R. X. Santos, S. C. Correia, C. Carvalho, M. S. Santos, I. Baldeiras, C. R. Oliveira and P. I. Moreira, Insulin-induced recurrent hypoglycemia exacerbates diabetic brain mitochondrial dysfunction and oxidative imbalance. Neurobiol Dis., 49, 2013a, $1-12$.

[9]. A. Maritim, B. A. Dene, R. A. Sanders and J. B. Watkins, Effects of pycnogenol treatment on oxidative stress in streptozotocininduced diabetic rats. J Biochem Mol Toxicol 17 (3), 2003, 193-199.

[10]. A. M. Berryman, A. C. Maritim, R. E. Sanders and J. B. Watkins, Influence of treatment of diabetic rats with combinations of pycnogenol, beta-carotine, and alpha-lipoic acid on parameters of oxidative stress. J. Bio. Chem. Mol. Toxicol., 18(6), 2004, 345352.

[11]. J. Busserolles, E. Gueux, B. Balasinska, Y. Piriou, E. Rock, Y. Rayssiguier and A. Mazur, In vivo antioxidant activity of procyanidrich extracts from grape seed and pine (Pine maritime) bark in rats. Int J vitam nutr res., 76(1), 2006, 22-27.

[12]. M. Dvorakova, M. Sivonova, J. Trebaticka, I. Škodáček, I. Waczuliková, J. Muchová and Z. Duračková, The effect of polyphenolic extract from pine bark, pycnogenol on the level of glutathione in children suffering from attention deficit hyperactivity disorder (ADHD). Redox Rep. 11(4), 2006, 163-172.

[13]. M. Kolacek, J. Muchova, S. Vrankova, L. Jendekova, O. Pechanova, C. Watala and Z. Duracova, Effect of natural polyphenols, pycnogenol on superoxide dismutase and nitric oxide synthase in diabetic rats. Prague med rep., 111(4), 2010, $279-288$.

[14]. B. Aydin, M. Unsal, Z. A. Secerogluo and Y. Gulbahar, The antioxidant and antigenotoxic effects of pycnogenol on rats treated with cisplatin. Biol. Trace. Elem. Res., 142, 2011, 638-650.

[15]. I. C. Lee, S. H. Kim, I. S. Shin, C. Moon, S. H. Park, S. H. Kim, S. C. Park, H. C. Kim and J. C. Kim, Protective Effects of Pine Bark Extract on Hexavalent Chromium-Induced Dermatotoxicity in Rats. Phytother. Res., 26 (10), 2012, 1534-1540.

[16]. K. Parveen, T. Ishrat, S. Malik, M. A. Kausar And W. A. Siddiqui, Modulatory effects of pycnogenol in a rat model of insulindependent diabetes mellitus: biochemical, histological, and immunohistochemical evidences. Protoplasma, 250 (1), $2013,347-360$.

[17]. P. Voss, L. Horakova, M. Jakstadt, D. Kiekebusch and T. Grune, Ferritin oxidation and proteasomal degradation: protection by antioxidants. Free Radic. Res., 40 (7), 2006, 673-683.

[18]. A. Eryilmaz, N. Eliyatkin, B. Demirci, Y. Basal, I. K. Omurlu, C. Gunel, S. Aktas, A, Toka and S. Basak, Protective effect of pycnogenol on cisplatin induced ototoxicity in rats. Pharmaceutical Biology, 54 (11), 2016, 2777-2781.

[19]. P. Trinder, Ann Clin Biochem., 6: 1969, 24-33.

[20]. M. A. Morales, A. J, Jabbagy and H. R. Terenizi, Mutations affecting accumulation of glycogen, Neurospora News, 1973, pp. 2024.

[21]. E. Beutler, O. Duron and M. B. Kelly, Lab Clin. Med., 61, 1963, 882

[22]. W. Habig, M. Pabst and W. Jakoby, J. Biol . Chem., 249, 1974, 7130-7139.

[23]. H. Aebi, Catalase in vitro. Methods in Enzymology, 105, 1984, 121-126. 
[24]. M. Nishikimi, N. Appaji and K. Yagi, The occurrence of superoxide anion in the reaction of reduced phenazine methosulfate and molecular oxygen. Biochem. Biophys. Res. Commun., 46, 1972, 849-854.

[25]. D. Koracevic, G. Koracevic, V. Djordjevic, S. Andrejevic and V. Cosic, Methods for the measurement of antioxidant activity in human fluids. J. Clin. Pathol., 54, 2001, 356-361.

[26]. A. H. B. Wu, Tietz clinical guide to laboratory test, 4th Ed., WB Saunders Company, 2006, 470.

[27]. H. Ohkawa, W. Ohishi and K. Yagi, Assay for lipid peroxides in animal tissues by thiobarbituric acid reaction. Anal. Biochem., 95, 1979,351

[28]. A. G. Gornal, C. T. Bardauill and M. M. David, Determination of Serum proteins by the mean of the Biuret reactions. Biochemistry. $177,1949,751-766$.

[29]. P. E. Cryer, L. Axelrod, A. B. Grossman, S. R. Heller, V. M. Montori, E. R. Seaquist and F. J. Service, Evaluation and management of adult hypoglycemic disorders: an Endocrine Society Clinical Practice Guideline. J Clin Endocrinol Metab; 94, 2009,709

[30]. S. K. Bhardwaj, M. L. Sharma, G. Gulati, A. Chhabra, R. Kaushik, P. Sharma and G. Kaur Effect of starvation and insulin-induced hypoglycemia on oxidative stress scavenger system and electron transport chain complexes from rat brain, liver, and kidney. Mol Chem Neuropathol. 34 (2-3), 1998, 157-168.

[31]. A. Guyton and J. A. Hall, Textbook of Medical Physiology. (12 ${ }^{\text {th }}$ edition Saunders, an imprint of Elsevier Inc.1600 John F. Kennedy Blvd. St. 1800 Philadelphia, PA 19103-2899, 2011) PAGE 939-950.

[32]. P. Singh, A. Jain and G. Kaur, Impact of hypoglycemia and diabetes on CNS: Correlation of mitochondrial oxidative stress with DNA damage. Mol Cell Biochem; 1-2, 2004, 153-159.

[33]. E. Hassoun, J. Vodhanel, B. Holden and A. Abushaban, The effects of ellagic acid and vitamin E succinate on antioxidant enzymes activities and glutathione levels in different brain regions of rats after subchronic exposure to TCDD. J Toxicol Environ Health A; 5, 2006, 381-393.

[34]. S. J. Won, B. H. Yoo, T. M. Kauppinen, B. Y. Choi, J. H. Kim, B. G. Jang, M. W. Lee, M. Sohn, J. Liu, R. A. Swanson and S. W. Suh, Recurrent/moderate hypoglycemia induces hippocampal dendritic injury, microglial activation, and cognitive impairment in diabetic rats. Journal of Neuroinflammation, 22(9), 2012, 182-194.

[35]. S. Cardoso, S. C. Correia, R. X. Santos, C. Carvalho, E. Candeias, A. I. Duarte, A. I. Plácido, M. S. Santos and P. I. Moreira, Hyperglycemia, hypoglycemia and dementia: role of mitochondria and uncoupling proteins. Curr Mol Med., 13 (4), 2013b, 586601 .

[36]. M. Sivonová, I. Waczulíková, E. Kilanczyk, M. Hrnèiarová, M. Bryszewska and B. Klajnert, The effect of pycnogenol on the erythrocyte membrane fluidity. Gen Physiol Biophys., 23 (1), 2004, 39-51.

[37]. T. Grimm, A. Schafer and P. Hogger, Antioxidant activity and inhibition of matrix metalloproteinases by metabolites of maritime pine bark extract. Free Radic Biol Med., 36 (6), 2004, 811-822.

[38]. Y. S. Yang, T. H. Ahn, J. C. Lee, C. J. Moon, S. H. Kim, W. Jun, S. C. Park, H. C. Kim and J. C. Kim, Protective effects of pycnogenol on carbon tetrachloride-induced hepatotoxicity in Sprague-Dawley rats. Food and Chemical Toxicology, 46 (1), 2008 , $380-387$.

[39]. G. Edrees, M. EI-Sawi and M. El-Harty, Antioxidant defense of pycnogenol against glycerol induced acute renal failure in mice. The Egyptian Journal of Hospital Medicine, 34, 2009, 1-8.

[40]. K. Parveen, M. R. Khan, M. Mujeeb and W. A. Siddiqui, Protective effects of pycnogenol on hyperglycemia-induced oxidative damage in the liver of type 2 diabetic rats. Chem Biol Interact., 186 (2), 2010, 219-227.

[41]. B. M. Erben, H. S. Benjamin and M. D. Lau, Pycnogenol inhibits generation of inflammatory mediators in macrophages. Nutri. Research, 20 (2), 2000, 249-259. 